Originalni naučni rad

UDK 821.133.1.09-6 Diderot D.

Primljen: 6. marta 2021.

Prihvaćen: 19. aprila 2021.

Nermin S. Vučelj ${ }^{1}$

https://doi.org/10.46630/phm.13.2021.04

Université de Niš

Faculté de philosophie ${ }^{2}$

Département de langue et littérature françaises

\title{
DIDEROT ÉPISTOLIER SUR LAAFAIRE CALAS ET SUR LA DISSOLUTION DE L'ORDRE DES JÉSUITES
}

Le propos de cette recherche est d'analyser comment Denis Diderot aborde les questions religieuses dans son échange épistolaire. Lédition de la Correspondance (1997) établie par Laurent Versini a servi d'instrument de travail. La problématique du religieux figure dans soixante-sept lettres que Diderot a écrites à Sophie Volland, Voltaire, Damilaville, Vialet, d'Alembert, et à son frère, le prêtre Didier-Pierre Diderot. Le présent article se concentre sur les deux thèmes majeurs figurant dans la correspondance diderotienne : le premier - l'affaire Calas et l'entreprise de Voltaire contre l'" Infâme » dans le procès de Toulouse, le second - la dissolution de la Compagnie de Jésus en France. Lanalyse nous mène à la conclusion que Diderot épistolier dénonce des absurdités de religion étant dangereuses pour la société et souligne la nécessité de l'esprit critique, qui est celui de la philosophie des Lumières, et l'importance de l'Encyclopédie pour éclairer les citoyens.

Mots-clés : religion, les Calas, Ordre des jésuites, échange épistolaire, pensée critique

\section{Introduction}

Denis Diderot et le Bon Dieu se croisent souvent dans l'œuvre de l'auteur français de l'époque des Lumières. Les questions concernant la métaphysique de la Foi, la pratique religieuse, la politique de l'Église et la morale chrétienne figurent dans la fiction littéraire de Diderot, dans ses traités philosophiques et dans ses articles encyclopédiques. Comme, d'après les mots de Diderot, son tic était de moraliser et qu'il n'aurait rien eu à dire si l'on lui avait imposé le silence sur la religion et le gouvernement, il allait de soi que notre philosophe abordait le sujet du religieux dans sa correspondance aussi.

La problématique du religieux figure dans soixante-sept lettres que Diderot a écrites, en premier lieu à Sophie Volland, son amie et son grand

1 nermin.vucelj@filfak.ni.ac.rs

2 Cet article est rédigé dans le cadre du projet scientifique international Les langues, les littératures et les cultures romanes et slaves en contact et en divergence, $\mathrm{N}^{\circ}$ 1001-13-01, approuvé le $1^{\text {er }}$ mars 2021 par la Faculté de Philosophie de l'Université de Niš. 
amour, presque la moitié (30 lettres) ; ensuite, au «maudit saint » de son frère, le prêtre Didier-Pierre Diderot (9), à sa sœur Denise (7), au " patriarche de Voltaire » (3) ; suivent, au nombre de deux, les lettres à Damilaville, à Vialet et au père Berthier dirigeant les Mémoires de Trévoux ; et une lettre à chacun des destinataires suivants: d'Alembert, Falconet, Grimm, Malesherbes, Jacques-Henri Meister, les médecins Tronchin et Clerc, le père Castel, le comédien David Garrick, la princesse russe Catherine Dashkoff et l'impératrice Catherine II. Il reste à noter une lettre adressée « aux parents et amis de Langres » pour compléter la liste de soixante-sept lettres relatives à la problématique du religieux, adressées à dix-neuf destinataires au total. ${ }^{3}$

Dans son échange épistolaire abordant le sujet du religieux, Diderot se préoccupe de l'affaire Calas et de l'entreprise de Voltaire contre l' "Infâme " dans le procès de Toulouse ; il fait des commentaires sur l'interdiction de l'Ordre des jésuites au Portugal et en France ; en philosophe, il dénonce des absurdités de religion, dangereuses pour la société, et souligne l'importance de sa mission encyclopédique. D'autres sujets y figurent, à travers quelques phrases en marge du sujet principal de la lettre, dans deux ou trois courts paragraphes, à savoir en alinéas. Le présent article dégage les deux thèmes particuliers dans les lettres de Diderot : le calvaire de la famille Calas à Toulouse et la destruction de la Compagnie de Jésus en France.

\section{Laffaire Calas}

Selon Jean M. Goulemot (Goulemot 2001 : 79), c'est «dans une même saisie archéologique des intellectuels » que l'on confond l'affaire Callas et l'affaire Dreyfus. Comme Goulemot le souligne, à la différence d'Émile Zola nommant les responsables du procès politique contre Dreyfus, Voltaire «n'est pas en règle générale un pamphlétaire dénonciateur » (2001: 80), et il laisse le soin à un avocat spécialisé de présenter un mémoire en réhabilitation auprès du Conseil du roi. Sans nommer les juges toulousains Voltaire "ne dénonce que les irrégularités de la procédure, le fanatisme sectaire des pénitents et l'ambiance de guerre religieuse qui règne encore dans la ville où les protestants sont mal tolérés » (2001 : 79-80). La conclusion de Goulemot est-elle vraiment soutenable? Voltaire dénonce-t-il des irrégularités procédurales sans montrer du doigt les juges du procès toulousain ? Car, ce défenseur des Calas déclare que les huit juges de Toulouse peuvent faire brûler son Traité sur la tolérance et nous rappelle que l'on avait déjà brûlé les Lettres provinciales de Pascal (VoL-

3 La Correspondance de Diderot (Robert Laffont, 1997) établie par Laurent Versini sert d'instrument de travail pour cette recherche. Lédition contient 626 lettres, ce qui fait les quatre-cinquièmes de la totalité des lettres conservées dont Versini exclut celles d'attribution douteuse et celles de contenu banal et informatif. La totalité de la Correspondance a été étudiée dans cette recherche pour y dégager les lettres relatives à la religion. 
TAIRE $1989: 151)$.

Néanmoins, des parallèles entre deux affaires politiques s'imposent. Le cas d'Alfred Dreyfus a bouleversé la société française de la Troisième République à la fin du XIX ${ }^{\mathrm{e}}$ siècle. De même, durant la période des années 1760 , selon Arthur Wilson « la conscience de chaque Français fut torturée par l'affaire Calas » (WiLson 1985 : 367). Émile Zola a alarmé l'opinion publique par sa lettre «J'accuse » publiée dans le journal Aurore, et Voltaire n'a pas cessé, pendant trois ans, de " harceler l'esprit et la conscience des Français » (1985: 367). Sylvain Menant souligne qu'au sujet de l'affaire Calas «Voltaire agit en journaliste engagé », que nous pouvons suivre toute l'affaire, jusqu'à la révision et la réhabilitation, à travers sa production, car « il ne cesse de tenir ses lecteurs au courant par des appendices, des allusions, des digressions » et qu' " il a crée dans notre civilisation un type d'écrivain engagé destiné à un grand avenir » (Menant 1977 : 137-138).

Dans l'introduction de son édition du Traité sur la tolérance, René Pomeau constate que Voltaire a choisi, comme point de départ de sa campagne contre la persécution religieuse, ce drame de l'intolérance se déroulant à Toulouse en 1761 et 1762, et suggère, lorsque nous lisons le Traité sur la tolérance, de nous remémorer « cette ambiance de l'ancienne France, où le pouvoir s'arrogeait très normalement le droit de tourmenter des hommes pour leurs croyances » (POMEAU 1989 : 8). Rappelons-nous que les autorités se sont efforcées de prouver que le jeune homme Marc-Antoine Calas sétait secrètement converti au catholicisme et que, pour cette raison, sa famille calviniste l'a assassiné le 13 octobre 1761. Jean Calas, le père du jeune homme suicidé, ${ }^{4}$ a été condamné à mort le 9 mars 1762, et exécuté le lendemain par le supplice de la roue.

Jean Pomeau constate que l'enquête judiciaire «s'orienta dans une seule direction, qui s'avéra une impasse : le crime calviniste » et que " les sentiments d'intolérance furent ici déterminants » (1989: 15). Il fallait que toute la famille fût coupable. Le procureur a requis pour le père et son fils Pierre la mort par le supplice de la roue, pour la mère la mort par pendaison. Mais le tribunal n'a pas osé aller si loin. Jean Calas à été condamné à être rompu vif et le fils Pierre a été condamné au bannissement. Les autres inculpés ont été acquittés. Selon Pomeau, "cétait reconnaître implicitement l'erreur judiciaire » (1989 : 17). Voltaire étudiait le cas des Calas pendant trois mois et il a pris l'affaire en main. En 1763 il a distribué son Traité sur la tolérance aux ministres d'État, à $\mathrm{M}^{\mathrm{me}}$ de Pompadour, au roi de Prusse, à des princes d'Allemagne. Selon

4 René Pomeau explique que c’est le cadet, Pierre Calas, qui a trouvé son frère aîné mort dans le magasin de tissus de leur père, pendu à une corde fixée à un rouleau de bois, destiné à enrouler les étoffes. Ce pauvre suicidé avait-il perdu de l'argent de son père au jeu ou autrement, et sétait-il donné la mort à cause de cela ? Ou, un assassin l'avait-il guetté pour le voler ou pour une autre raison? Nous ne le saurons jamais. (PomEAu 1989 : 15). 
Pomeau, "c'est devant l'Europe des lumières qu'il plaide en appel la cause des Calas, et qu'il va gagner» $(1989: 18)$.

Arthur Wilson trouve $(1985: 367)$ que Diderot était « étonnamment réservée » dans cette agitation générale causée par l'affaire Calas, car il n’a pris aucune position publique, et que nous pouvons le regretter. Daniel Mornet constate que Diderot « reste étranger à toutes les polémiques, se garde de tous les scandales» (Mornet 1941: 76), en nous suggérant la conclusion que le premier encyclopédiste ne voulait pas compromettre son entreprise éditoriale. Pour soutenir son hypothèse, Mornet se réfère à la situation défavorable dans laquelle s'est trouvé le projet encyclopédique : en 1759, le pape Clément VIII a publié une condamnation de l'Encyclopédie ou Dictionnaire raisonné des sciences, des arts et des métiers; en 1760, Le Franc de Pompignan a prononcé à l'Académie française un violent réquisitoire contre les philosophes; Palissot a mis sur scène Les Philosophes ridiculisant les encyclopédistes. En outre, Le Breton a corrigé les épreuves déjà revues par Diderot et a supprimé tout ce que lui paraissait capable d'inquiéter l'autorité. Pour cette raison Mornet juge que «les tomes imprimés après le tome VII ne sont pas la vraie Encyclopédie » (1941 : 76). Malgré sa colère contre cette décision, le premier encyclopédiste s’est finalement résigné.

À la nouvelle de la dissolution de l'Ordre des jésuites, des mots de soulagement que Diderot a exprimé se trouvant « délivré d'un grand nombre d'ennemis puissants » (Diderot 1997 : 407) contredisent-ils à la thèse de Mornet sur la prudence du Philosophe à l'égard de l'affaire Calas ? Comme les deux procès étaient presque simultanés, et que le cas des jésuites a touché à sa fin bien avant le cas des Calas, on pourrait se poser la question suivante : étant donné que Diderot sétait enthousiasmé par la destruction des jésuites, n’aurait-il pas été encouragé de soutenir plus ouvertement la défense des Calas?

Bien informé sur l'affaire, Diderot épistolier se procurait des papiers concernant le procès de Toulouse et n'a pas manqué d’admirer Voltaire et de le soutenir dans son entreprise. À propos du rôle que ce patriarche des philosophes a joué dans la défense des Calas, Diderot écrit à Sophie Volland: "Quand il y aurait un Christ, je vous assure que de Voltaire serait sauvé.» (1997 : 406). En élaborant les raisons pour lesquelles Voltaire écrivait et agissait en faveur de la famille Calas, Diderot a affirmé que de Voltaire (qu'il nommait toujours en lui ajoutant la particule « de ») avait de l'âme, de la sensibilité, que l'injustice le révoltait et qu'il sentait l'attrait de la vertu. Or, défendre les Calas, cétait « le bel emploi du génie » $(1997: 406) .{ }^{5}$

5 Dans Le Neveu de Rameau, Diderot fait hommage à Voltaire pour son rôle dans l'affaire Calas. L'interlocuteur nommé Moi déclare : "C'est un sublime ouvrage que Mahomet; j’aimerais mieux avoir réhabilité la mémoire des Calas " (Diderot 2004 : 613). Cette phrase, plus explicite par le sens, voire plus claire, sera reprise dans l'Essai sur les règnes de Claude et de Néron, comme nous le signale Michel Delon dans ses notes au Neveu de Rameau (2004 : 1158). 
Chronologiquement, c'est la première note sur l'affaire Calas dans la Correspondance. Signée le 8 août 1762, cette lettre à Sophie Volland prouve que le sujet a été abordé dans l'échange épistolaire diderotien bien avant la date indiquée, car Diderot y a annoncé qu'il enverrait à son amie « deux nouveaux papiers sur l'affaire Calas » (DIDEROT 1997 : 406), ce qui signifie qu'ils avaient déjà vivement discuté sur cette affaire. Laurent Versini, le directeur de l'édition de la Correspondance, nous explique que les deux documents en question dans cette lettre sont des Mémoires de Donat Calas pour son père, sa mère et son frère écrit par Voltaire, et une Déclaration de Pierre Calas des 22 et 23 juillet 1762. Il est probable que ces mêmes documents soient en question lorsque Diderot a annoncé à sa "bonne et tendre amie » (le 15 août 1762) qu'il avait " une foule de choses intéressantes » à lui envoyer : " la suite des papiers sur les Calas, l'Éloge de Crébillon, etc. » (1997 : 412).

Une semaine plus tard (le 22 août), notre épistolier espère encore « joindre à cette lettre la suite de l'affaire tragique des Calas » (1997 : 416), car l'impression n'en était pas achevée. Qu'il s'agit toujours des mêmes documents que Diderot a promis d'envoyer à Sophie Volland dans les trois lettres ici mentionnées prouve la lettre du 5 septembre, dans laquelle l'épistolier explique qu'il attend "deux nouveaux papiers de Voltaire sur les Calas », qu' " ils seront suivis d'une consultation d'avocats, d'un mémoire, de la requête en cassation » (1997 : 432) et qu'il promet à son amie de les avoir tout. Les documents finalement arrivés, et après les avoir examinés, le Philosophe a consacré la moitié de sa lettre écrite du 30 septembre à la problématique du procès de Toulouse. Il se demande « à quoi sert une vie passée avec honneur, si elle ne nous protège pas contre les attaques de la méchanceté et le soupçon d'un crime » (1997: 451) et où est la distinction entre l'homme de bien et le scélérat.

En envisageant la défense possible devant les juges, Diderot soulève un paradoxe en plaidant pour la cause des Calas. Si Jean Calas avait tué son fils de crainte qu'il ne changeât de religion, il aurait été un fanatique regardant son crime comme une action héroïque ; or, il aurait dû défendre sa cause devant les juges. Mais, comme il proteste de son innocence, «s'il est coupable, il ment à la

Voici la phrase entière tirée du premier tome de l'édition CEuvres établie par Versini : «Parmi les idées de Sénèque, je me plais encore plus à citer celles qui montrent la bonté de son âme que celles qui montrent la beauté de son esprit, parce que je fais plus de cas de l'une de ces qualités que de l'autre, parce que j'aimerais mieux avoir fait une belle action qu'une belle page, parce que c'est la défense des Calas et non la tragédie de Mahomet que j’envierais à Voltaire. » (DidEROT $1994: 1170$ )

Dans son article « Voltaire et Rousseau dans les lettres de Diderot » („Волтер и Русо у Дидроовим писмима"), en constatant bien juste que Diderot et Voltaire nétaient pas de vrais amis, Milan Janjić conclut que Voltaire représentait pour Diderot « un modèle spirituel dans sa lutte contre la superstition religieuse » (Волтер ће Дидроу бити више духовни узор у борби против религијског сујеверја”). (JaNJIć 2020: 445) 
face du ciel et de la terre »; or, « il en a donc perdu le mérite en la désavouant lâchement » (1997: 452). S'il nie ce qu'il a fait, il n'est pas un vrai croyant, « il est donc athée ; il en a le discours » (1997: 452). S’il est athée, il n’est plus fanatique, et il ne pouvait pas tuer son fils, car étant athée " pourquoi, contempteur de tout dieu et de tout culte, aurait-il tué son fils pour en avoir voulu prendre un autre que celui dans lequel il était né » (1997: 452). Notre épistolier voit « dans cette cause cent moyens secret que les avocats ni de Voltaire n'ont point aperçus » (1997 : 452). Il affirme que ces arguments pourraient servir à un avocat étant maitre de l'art de la parole.

Après cette lettre de la fin de septembre 1762, Diderot ne revient au cas des Calas qu'après trois ans. Dans l'entre-temps, il n'y avait que trois lignes ajoutées dans sa lettre à Damilaville (du 5 mars 1764) pour le rappeler qu'il lui avait demandé vingt-cinq exemplaires du Traité sur la tolérance de Voltaire. Chronologiquement, les dernières notes que nous trouvons sur l'affaire Calas dans la Correspondance de Diderot sont celles dans deux lettres datant du septembre 1765. Chez le peintre Jean-Louis Vanloo Diderot a rencontré M. Ramsay, le premier peintre du roi d'Angleterre, mentionné dans l'Histoire d'Elizabeth Canning figurant comme une des pièces du dossier Calas. Diderot explique à Sophie Volland (lettre du 8 septembre 1765) que Voltaire se réfère à l'histoire d'une jeune fille dont la fourberie avait exposé sept honnêtes gens à périr ignominieusement, mais qui ont été sauvés grâce à Ramsay dont les arguments inébranlables ont convaincu les juges de l'innocence des gens faussement accusés. Quatre jours plus tard, dans la seconde lettre de septembre (du 12 septembre 1765), Diderot a confirmé à Damilaville qu'il n’y avait plus d'obstacle pour la souscription en faveur de la famille Calas et que Grimm et lui devaient mettre leur signature. ${ }^{6}$ Cétait la dernière mention épistolaire de Diderot relative au cas des Calas.

\section{La destruction des jésuites en France}

Dans l'appendice du Traité sur la tolérance, intitulé « Article nouvellement ajouté, dans lequel on rend compte du dernier arrêt rendu en faveur de la famille Calas ", Voltaire remarque que la grande affaire des jésuites « fit

6 Il s'agit de la souscription pour une Estampe tragique et morale représentant la famille Calas, dessinée par Carmontelle et gravée par Delafosse, dont le bénéfice devait en aller à la famille Calas. Ce projet du soutien public à la famille Calas représentait, selon Diderot, « la marque la plus évidente du progrès de la raison et des services de la philosophie » (1997: 518). Dans sa lettre pour Sophie (le 18 août 1765), notre épistolier considérait la liste des sous-scripteurs comme «le monument le plus honorable de la bienfaisance naturelle» (1997: 519). Mais, le projet a affronté des obstacles pour un certain temps et Diderot y voyait la vengeance la plus cruelle du parlement de Toulouse et «le témoignage le plus authentique du mépris qu’on porte à présent à ces opinions religieuses qui ont si souvent étouffé l'humanité dans le cœur de l'homme» (1997: 518). 
pendant plusieurs mois perdre de vue au public le procès des Calas » et recommande de voir l'histoire de leur proscription dans « l'excellent livre » de Jean Le Rond d'Alembert, " ouvrage impartial, parce qu'il est d'un philosophe ", écrit " avec une supériorité de lumières" (VoltaIre 1989: 154). Dans une courte lettre que Versini date «vers le 10 mai 1765 » Diderot a envoyé son compliment à d'Alembert pour son traité Sur la destruction des jésuites en France, qu'il avait lu avec un grand plaisir, en le remerciant pour cet ouvrage écrit "pour l'honneur de la philosophie» (Diderot 1997 : 490).

Un bref rappel : le parlement a profité d'une excellente occasion pour détruire les jésuites, celle de la banqueroute de La Valette entrainant dans sa ruine des banquiers de Marseille. Comme la Compagnie de Jésus avait refusé de rembourser les créanciers et avait préféré porter l'affaire devant le parlement de Paris, celui-ci a entrepris contre les jésuites des procédures aboutissant à la suppression de leur ordre en France. ${ }^{7}$ Dans la destruction de « ces grands grenadiers du fanatisme » et « les plus dangereux ennemis de la raison», comme d'Alembert les nomme, il voit en premier lieu la victoire des philosophes et des jansénistes : "Cest proprement la philosophie qui, par la bouche des magistrats, a porté l'arrêt contre les Jésuites ; le jansénisme n’en a été que le solliciteur.» (AlEmbert 1876: 126-127). Néanmoins, l'auteur népargne pas les critiques aux jansénistes, ce que Diderot souligne dans sa lettre en concluant que son ancien co-directeur de l'Encyclopédie devait se faire «la belle nuée d'ennemis secrets » (Diderot $1997: 490$ ).

Cette courte lettre à d'Alembert est chronologiquement la dernière dans laquelle Diderot traite le sujet de la dissolution des jésuites. La première lettre dans laquelle lépistolier aborde cette question est celle du 12 octobre 1761, mais il s'y agit du procès mené contre les jésuites au Portugal et de leur bannissement de ce pays. Diderot commence son compte rendu épistolaire pour Sophie Volland par des nouvelles de Lisbonne « que toutes les bouches de la ville [qui] l'annoncent» (1997: 363) : accusés de la tentative d'assassinat du roi de Portugal Joseph $\mathrm{I}^{\mathrm{er}}$, les vingt-sept jésuites et leurs conspirateurs, six Juifs et deux Français, ont été brûlés vifs. Cinq jours plus tard, Diderot s'exprime de navoir presque plus de courage d'écrire des nouvelles. Le Philosophe juge que le pouvoir du Portugal brûlera autant de jésuites qu'il voudra. Il faudrait voir ce commentaire de Diderot dans le contexte de la vraie chasse aux sorcières que le pouvoir du Portugal avait été lancée, ce qui a abouti à l'exécution des représentants de plusieurs familles nobles, condamnés pour haute trahison.

7 Après que les fameuses Constitutions de la Société de Jésus avait été livrées, pour la première fois en France, au grand jour de la publicité, elles étaient considérées comme hostiles à la monarchie, et les avocats des créanciers les ont utilisées comme la preuve que la Société était un tout indivisible et, par conséquent, le crime du Père La Vallette était celui de l'Ordre. C’est donc à la suite du procès contre La Valette que la Compagnie de Jésus a été supprimée. (Boucher $1846: 316$ ) 
C'est dans la lettre du 8 août 1762 où notre philosophe se réfère pour la première fois au procès des jésuites en France ainsi quà l'affaire Calas. Diderot annonce à Sophie Volland la grande nouvelle : le Parlement a condamné la Société de Jésus par l'arrêt du 6 août. ${ }^{8}$ Dans sa lettre suivante, du 12 août, notre épistolier a constaté avec soulagement qu'il serait désormais «délivré d'un grand nombre d'ennemis puissants » (1997 : 407). Les jésuites, dont le régime " n'est que le machiavélisme réduit en préceptes ", ont brouillé l'Église et l'État, et Diderot conclut que, «soumis au despotisme le plus outré dans leurs maisons, ils en étaient les prôneurs les plus abjects dans la société » (1997 : 407).

En lisant la lettre adressée à Sophie Volland, en date de 5 septembre 1762, nous apprenons que, pendant le souper réunissant Diderot, Grimm, Damilaville, le docteur Gatti et l'ex-jésuite l'abbé Raynal, « on causa beaucoup de l'affaire des jésuites, qui était toute fraîche " (1997 : 427). Cette lettre nous raconte aussi une anecdote sur Henri Griffet, prédicateur ordinaire du roi et aumônier à Vincennes et à la Bastille qui, selon la note de Versini (1997: 1382), plaidait vigoureusement pour la cause de la Compagnie de Jésus. Henri Griffet pleurait le sort des jésuites chassés, « dépouillés de leurs vêtements, de leur nom, de leur état, d'une maison où ils étaient entourés des cœurs de leurs rois », en faisant allusion au fait que l'église Saint-Louis-des-Jésuites possédait les cœurs de Louis XIII et Louis XIV. Son interlocuteur lui réplique : "Mon père, voilà ce que c'est que de sêtre un peu trop pressé d'avoir celui de Louis $\mathrm{XV} »(1997: 427)$.

Il faut se rappeler que l'attentat de Lisbonne a été précédé par l'attentat échoué à Paris. Selon Adolphe Boucher (Boucher 1846 : 299), si les Jésuites nétaient pas ceux qui avaient poussé secrètement Robert-François Damiens à tuer Louis $\mathrm{XV}$, cétaient eux que l'opinion publique a désigné comme ses complices et ses excitateurs. Diderot, semble-t-il, admirait l'enthousiasme de Damiens, lorsqu'il a écrit à Sophie Volland (en date d'octobre 1760) que l'on avait condamné cet homme à être déchiré avec des ongles de fer, arrosé d'un métal bouillant et démembré par des chevaux, pour avoir osé attenter la vie de son souverain, et qui, après avoir entendu cette sentence terrible, avait froidement dit que la journée serait rude. Diderot épistolier pose la question rhétorique - «le crime serait-il capable d'un enthousiasme que la vertu ne pourrait concevoir» (DIDEROT $1997: 255)$.

L'héritage politique des jésuites en France était prépondérant que, cinq ans après la dissolution de la Compagnie de Jésus, Diderot constate, dans la lettre au comédien anglais David Garrick (du 26 janvier 1767), qu’il est impossible de traiter dans le théâtre français les caractères les plus ridicules moines, religieuses, abbés, évêques, et que le dramaturge qui oserait le faire 8 Nous lisons dans la brochure de Jean d'Alembert que le premier arrêt, du 6 août 1761, impose la clôture du collège jésuite ; ce deuxième arrêt, du 6 août 1762, a condamné l'institut de l’Ordre et les jésuites ont été dissous et leurs biens aliénés. (Alembert 1876 : 111-114) 
« risquerait d’obtenir un logement aux dépens de l'État, à la Bastille ou à Bicêtre" (1997 : 722). Selon Diderot, une croix et un capuchon font une chose respectable en France, à la différence de l'Angleterre dont le théâtre peut représenter les membres du clergé comme des personnages négatifs.

\section{Les autres considérations relatives au religieux}

Les autres thèmes s'inscrivant dans le contenu religieux figurent en alinéas dans léchange épistolaire diderotien comptant soixante-sept lettres. Suivent de brèves notes dans l'ordre chronologique.

Diderot épistolier répond à Voltaire (le 11 juin 1749) sur son reproche d'avoir utilisé Saunderson dans la Lettre sur les aveugles pour prêcher l'athéisme. ${ }^{9}$ Dans la lettre du 20 octobre 1758, Diderot le dramaturge a appris à Malesherbes d'avoir supprimé les parties de la pièce Père de famille qui l'avaient offensé et d'avoir adouci celles qui lui avaient paru dures. En revanche, Diderot a défendu la scène dans laquelle le père donnait sa malédiction à son fils, car c'était la réaction d'un homme pieux, convenable au caractère du père de famille et à létat dans lequel il se trouvait. Bien plus tard, Diderot a averti Voltaire (le 29 septembre 1762) sur les difficultés de l'Encyclopédie hantée par «l'Infâme".

A Damilaville (le 12 septembre 1765) il a raconté la fable sur le misanthrope réfugié dans une caverne qui avait conçu la manière la plus terrible de se venger de l'espèce humaine : en blasphémant Dieu auquel les gens attachaient plus d'importance quà leur vie et sur lequel ils ne pouvaient jamais s'accorder. À propos de léloge des capucins de Vialet (juillet 1766), Diderot a dénoncé la religion chrétienne comme « la plus absurde et la plus atroce dans ses dogmes» et « la plus funeste à la tranquillité publique» (Diderot 1997 : 657). Il a appris à Falconet étant à Petersburg (mai 1768) sur les ennuis de librairies provoqués par «l'intolérance du gouvernement " s'accroissant de jour en jour dans le but déteindre les lettres et de ruiner le commerce de librairie. Il lui a découvert la riposte des philosophes par la contrebande de livres, et il a fait un commentaire sur la vente du Christianisme dévoilé d'Holbach.

Notre épistolier a écrit à la princesse Dashkoff (le 3 avril 1771) sur l'esprit du XVIII siècle, qui est celui de la liberté étant « l'assaut à la barrière de la religion " (1997 : 1067). Diderot a aussi fait un sermon évangélique dans une lettre à sa sœurette Denise (le 4 octobre 1771) et dans le même esprit lui a recommandé les pièces saintes de Racine dans une autre lettre (le 5 janvier 1772). Au médecin Clerc (le 8 avril 1774) il a annoncé son projet de publier en Russie les articles sur les matières religieuses dans l'Encyclopédie. À Meister (le

9 À Voltaire qui lui avait reproché de sêtre servi de l'aveugle-né Saunderson dans la Lettre sur les aveugles pour nier "l'Horloger» et pour fonder l'athéisme, le Philosophe a répondu qu'il croyait en Dieu quoiqu’il vît très bien avec les athées. (Diderot $1997: 15$ ) 
27 septembre 1780), il a écrit que La Religieuse représentait la contrepartie de Jacques le fataliste en la désignant comme " la plus effrayante satire des couvents » (1997 : 1309). De son retour de la Russie, où il avait passé cinq mois à la cour impériale, il a exprimé sa reconnaissance à Sa Majesté Catherine II (le 17 décembre 1774), en lui racontant sous forme de dialogue la conversation qu'il avait mené au sein de sa famille, en accentuant en particulier la réaction de l’impératrice à l'athéisme de l'Encyclopédiste.

\section{Conclusion}

De six cent vingt-six lettres de Diderot recueillies dans la Correspondance (1997), les soixante-sept sont relatives au religieux, à la théorie de la foi, à savoir à " la théologie, cette science de chimère ", comme Diderot la désigne dans ses Mélanges pour Catherine II (DiDerot 1995 : 265), et à la politique de l'Église, à savoir la pratique religieuse imprégnée de l'intolérance. La présente recherche s'est concentrée sur deux sujets dominants dans la correspondance diderotienne qui sont relatifs au religieux : l'affaire Calas et la suppression de l'Ordre des jésuites.

Dans son échange épistolaire, Diderot a dénoncé des absurdités de religion et a souligné l'importance d'une philosophie éclairée, car, comme il l'a affirmé dans sa lettre à Voltaire (le 29 septembre 1762), « la philosophie fait plus de gens de bien que la grâce suffisante ou efficace »; le vrai, le bon et le beau font une espèce de trinité de la Philosophie « qui vaut un peu mieux que la leur » (1997 : 449), à savoir la Sainte Trinité de la théologie catholique. Le prouve l'affaire judiciaire qui a fait périr une famille protestante, les Calas, pour un crime inexistant. Le prouve l'influence politique des membres d'une société qui prétendaient être compagnons de Jésus. Les activités dangereuses des jésuites ont été suspendues par la dissolution de leur ordre, qui était surtout hostile à la philosophie des Lumières et à l'entreprise encyclopédique. Si Denis Diderot n'a pas soutenu le procès des Calas par sa plume et devant le tribunal, comme Voltaire l'avait fait, et s'il n'a pas traité en philosophe la problématique de l'Ordre des jésuites comme d'Alembert l'avait élaboré dans son excellent traité, il n’est non plus resté étonnamment réservé ni étranger à toutes ces polémiques, comme Arthur Wilson et Daniel Mornet lui reprochaient. La présente recherche a essayé de le prouver en étudiant Denis Diderot en tant quépistolier traitant les deux grandes questions politico-religieuses de son époque, l'affaire Calas et la dissolution de la Compagnie de Jésus.

\section{Sources}

Alembert 1876: ALEMBERT, Jean Le Rond d'. Sur la destruction des jésuites en France. Paris : Librairie de la Bibliothèque Nationale, 1876. 
Diderot 2004: DIDEROT, Denis. Contes et romans. Michel Delon (éd.). Paris : Gallimard, Bibliothèque de la Pléiade, 2004.

Diderot 1997: DIDEROT, Denis. Euvres : Correspondance. Laurent Versini (éd.). Paris : Robert Laffont, 1995.

Diderot 1994: DIDEROT, Denis. Euvres : Philosophie. Laurent Versini (éd.). Paris : Robert Laffont, 1994.

Diderot 1995: DIDEROT, Denis. Euvres : Politique. Laurent Versini (éd.). Paris : Robert Laffont, 1995.

Voltaire 1989: VOLTAIRE, François-Marie Arouet, dit. Traité sur la tolérance. Paris : Flammarion, 1989.

\section{Références bibliographiques}

Boucher 1846: BOUCHER, Adolphe. Histoire dramatique et pittoresque des jésuites, $\mathrm{t}$. II. Paris : Éd, R. Prin, 1846.

Goulemot 2001: GOULEMOT, Jean M. Adieu les philosophes - que reste-t-il des Lumières. Paris : Éd. du Seuil, 2001.

Janjić 2020: JANJIĆ, Milan. „Volter i Ruso u Didroovim pismima”. Filolog, XXI. Banja Luka: Filološki fakultet, 2020, 434-452. [orig.] Јањић, Милан. „Волтер и Русо у Дидроовим писмима". Филолог, XXI. Бања Лука: Филолошки факултет, 2020, 434-452.

Menant 1977: MENANT, Sylvain. «Voltaire après 1750 ». In : Littérature française, t. X, Claude Pichois (éd.). Paris : Arthaud, 1977.

Mornet 1941: MORNET, Daniel. Diderot, l'homme et l'œuvre. Paris : Boivin et $C^{\text {le, }}$ 1941.

Pomeau 1989: POMEAU, René. «Introduction ». In : Voltaire, Traité sur l'intolérance. Paris : Flammarion, 1989.

Wilson 1985: WILSON, Arthur. Diderot, sa vie et son œuvre. Paris : Laffont-Ramsay, 1985. 
Нермин С. Вучељ

\section{ЕПИСТОЛАРНИ ДИДРО О АФЕРИ КАЛАС И О УКИДАҢУ ЈЕЗУИТСКОГ РЕДА}

Намера овог рада је да се анализира како је Дидро у својој преписци разматрао питања која се тичу религије. Француско издање Преписке (Correspondance, 1997), које је приредио Лоран Версини, представља основни истраживачки корпус. Религијска проблематика присутна је у шездесет седам писама које је француски философ послао Софији Волан, Волтеру, Дамилавилу, Вијалеу, Д’Аламберу и свом брату свештенику. Овај рад је усредсређен на две велике религијске теме присутне у Дидроовој преписци: прва је афера Калас и у вези с тим Волтерова улога у борби против „Бестиднице”, како је Цркву називао овај бранилац породице Калас, а друга је укидање Реда језуита у Француској. Анализа нас води до закључка да епистоларни Дидро разобличује религијску ускогрудост и нетрпељивост које су опасне по друштво и подвлачи значај које за грађанско просвећивање имају његова енциклопедијска мисија и критичка мисао која је суштина философије просветитељства.

Кључне речи: религија, породица Калас, Ред језуита, преписка, критичка мисао 